\title{
Optimal Design of Water Distribution Networks by Discrete State Transition Algorithm
}

\author{
Xiaojun Zhou David Yang Gao, and Angus R. Simpson
}

\begin{abstract}
Optimal design of water distribution networks, which are governed by a series of linear and nonlinear equations, has been extensively studied in the past decades. Due to their NP-hardness, methods to solve the optimization problem have changed from traditional mathematical programming to modern intelligent optimization techniques. In this study, with respect to the model formulation, we have demonstrated that the network system can be reduced to the dimensionality of the number of closed simple loops or required independent paths, and the reduced nonlinear system can be solved efficiently by the Newton-Raphson method. Regarding the optimization technique, a discrete state transition algorithm (STA) is introduced to solve several cases of water distribution networks. In discrete STA, there exist four basic intelligent operators, namely, swap, shift, symmetry and substitute as well as the "risk and restore in probability" strategy. Firstly, we focus on a parametric study of the restore probability $p_{1}$ and risk probability $p_{2}$. To effectively deal with the head pressure constraints, we then investigate the effect of penalty coefficient and search enforcement on the performance of the algorithm. Based on the experience gained from the training of the Two-Loop network problem, the discrete STA has successfully achieved the best known solutions for the Hanoi and New York problems. A detailed comparison of our results with those gained by other algorithms is also presented.
\end{abstract}

Index Terms-Discrete state transition algorithm, water distribution network, intelligent optimization, NP-hardness.

\section{INTRODUCTION}

$\mathbf{P}$ IPES, hydraulic devices (pumps, valves, etc.) and reservoirs are connected in a water distribution network in a complex manner. The physical behavior of a looped network is governed by a set of linear and nonlinear equations, including continuity and energy equations, and head loss functions. The overall planning tasks to be performed in water distribution networks consists of three kinds of problems: layout, design and operation. Although these problems are not independent with each other, they can be formulated and solved separately from a technical point of view since each one can be considered as a parameter when others are being solved. In this work, we focus on the optimal design problem.

Optimal selection of pipe diameters to constitute a water distribution network respecting certain pressure requirements has

Xiaojun Zhou is with the School of Science, Information Technology and Engineering, University of Ballarat, Victoria 3353, Australia and School of Information Science and Engineering, Central South University, Changsha 410083, China (tiezhongyu2010@gmail.com)

David Yang Gao is with the School of Science, Information Technology and Engineering, University of Ballarat, Victoria 3353, Australia (d.gao@ballarat.edu.au)

Angus R. Simpson is with the Centre for Applied Modeling in Water Engineering, School of Civil and Environmental Engineering, University of Adelaide, Adelaide, SA 5005, Australia (angus.simpson@adelaide.edu.au)

Manuscript received **, 2013; revised **, 2013. been shown to be an NP-hard problem [1], mainly due to two reasons: nonlinear equations and discrete-valued diameters. A terribly clumsy method for designing pipe network is by enumeration or complete trial and error [2]. Traditional methods are to linearize and relax the problem firstly to facilitate the use of linear programming and nonlinear programming, and then they have to round off the solution to the nearest discrete diameters [3]-[7]. Such algorithms can not guarantee global optima and sometimes cause infeasible solutions. In the last few decades, intelligent optimization techniques including: genetic algorithm [8]-[10], simulated annealing [11], shuffled complex evolution [12], ant colony optimization [13], [14], harmony search [15], particle swarm optimization [16], differential evolution [17] and some of their hybrids [18], [19], have found wide applications in this field. The advantages of using these stochastic algorithms are: (1) simple representation of a discrete-valued solution; (2) independent to the problem structure to some extent; (3) easy computation due to the only use of the information about the objective function; (4) high probability to gain the global optimum or approximate global optimum in a reasonable amount of time.

We introduce the recently developed intelligent optimization algorithm, state transition algorithm (STA) [20]-[22], which shows fantastic performance in continuous function optimization. In [23], a discrete STA was proposed to solve the traveling salesman problem, and the results demonstrated that it consumed much less time and had better search ability than the well-known simulated annealing and ant colony optimization. The goal of this paper is to apply the discrete STA to the optimal design problem of the water distribution networks.

This paper is organized as follows. In Section II, the optimization model of water distribution networks is established, including the objective function, decision variables and some constraints. In Section III, the basic key elements in discrete STA are introduced. It focuses on the intelligent operators of discrete STA and a parametric study of the "restore probability" and "risk probability" is the emphasis. How to deal with the constraints and the implementation of the discrete STA for the optimal design problem are illustrated in Section IV. In Section V, several case studies are given. The TwoLoop network is mainly studied to investigate the effect of penalty coefficient and search enforcement on the performance of the discrete STA. The gained experience is applied to other cases and the results achieved by the proposed discrete STA with other optimization algorithms are presented as well. Conclusion is derived in Section VI. 


\section{OPTIMIZATION MODEL FORMULATION OF WATER DISTRIBUTION NETWORKS}

For a given layout of pipes and a set of specified demand patterns at the nodes, the optimal design of a water distribution network is to find the combination of commercial pipe sizes which gives the minimum cost, subject to the following constraints:

- continuity of flow;

- head loss;

- conservation of energy;

- minimum pressure head.

\section{A. The objective function}

Considering that the pipe layout, connectivity and imposed minimum head constraints are known, in the optimal design problem of the water distribution network, the pipe diameters are the only decision variables. As a result, the objective function is assured to be a cost function of pipe diameters

$$
\min _{D_{j} \in \Omega} f_{o b j}=\sum_{j=1}^{N P} L_{j} c\left(D_{j}\right)
$$

where, $\Omega$ is a set of commercial pipe sizes, $N P$ is the number of pipes, and $L_{j}$ is the length of pipe $j$, which is known in this study. $c\left(D_{j}\right)$ indicates that for every commercial pipe size, there is a corresponding cost per unit associated with it.

\section{B. Continuity equation}

Conservation of mass at nodes or junctions in a water distribution network yields a set of linear algebraic equations in terms of flows. At each node, flow continuity should be satisfied,

$$
-\sum Q_{i n}+\sum Q_{o u t}+D M=0,
$$

where, $D M$ is the demand at the node, $Q_{i n}$ and $Q_{o u t}$ are the flow entering and leaving the node, respectively.

\section{Head loss equation}

The head loss in a pipe in the water distribution network can be computed from a number of empirically obtained equations. The two commonly used equations are the Darcy-Weisbach head loss equation and the Hazen-Williams head loss equation. The general expression for the head loss in a pipe $j$ located between nodes $i$ and $k$ is given by

$$
H_{i}-H_{k}=r_{j} Q_{j}\left|Q_{j}\right|^{\alpha-1}=\omega \frac{L_{j}}{C^{\alpha} D_{j}^{\beta}} Q_{j}\left|Q_{j}\right|^{\alpha-1},
$$

where, $H_{i}$ and $H_{k}$ are nodal pressure head at the end of the pipe at node $i$ and $k$ respectively; $r_{j}$ is called resistance factor for the pipe $j ; Q_{j}$ is the flow in pipe $j ; \omega$ is a numerical conversion constant depending on the units used; $L_{j}$ is the length of pipe $j ; C$ is the roughness coefficient; $\alpha$ and $\beta$ are coefficients.

For International System of Units (SI), $\omega=10.6744$ or $\omega=$ $10.5088, \alpha=1 / 0.54=1.852$ and $\beta=2.63 / 0.54=4.871$ are employed in this study using the Hazen-Williams formula.

\section{Energy equation}

Energy conservation equations around closed simple loops or between fixed head nodes along required independent paths in a network are nonlinear. Upon traversing a closed simple loop or a required independent path, the sum of pipe head losses around the loop or the path must be zero, which can be expressed as

$$
\sum_{j \in L_{s}} \omega \frac{L_{j}}{C^{\alpha} D_{j}^{\beta}} Q_{j}\left|Q_{j}\right|^{\alpha-1}-\sum_{j \in L_{s}} E L_{j}=0,
$$

where, $L_{s}$ is the indices of pipes in a closed simple loop or a required independent path; $E L_{j}$ is the hydraulic grade line at the reservoir $j$.

\section{E. Minimum pressure head}

The minimum pressure head constraints at each node are given as follows

$$
H_{i} \geq H_{i \min }, \forall i=1, \cdots, N J
$$

where, $H_{i \min }$ is known, and $N J$ is the number of nodes.

\section{A BRIEF REVIEW OF THE DISCRETE STATE TRANSITION ALGORITHM}

Let's consider the following unconstrained integer optimization problem

$$
\min f(x) \text {, }
$$

where, $x=\left(x_{1}, \cdots, x_{n}\right), x_{i} \in \mathcal{I} \subset \mathcal{Z}^{m}, i=1, \cdots, n$, and $f(x)$ is a real-valued function.

\section{A. The framework of the discrete state transition algorithm}

If a solution to a specific optimization problem is described as a state, then the transformation to update the solution becomes a state transition. Without loss of generality, the unified form of discrete state transition algorithm can be described as

$$
\left\{\begin{array}{l}
x_{k+1}=A_{k}\left(x_{k}\right) \bigoplus B_{k}\left(u_{k}\right) \\
y_{k+1}=f\left(x_{k+1}\right)
\end{array},\right.
$$

where, $x_{k} \in \mathcal{Z}^{n}$ stands for a current state, corresponding to a solution of a discrete optimization problem; $u_{k}$ is a function of $x_{k}$ and historical states; $A_{k}(\cdot), B_{k}(\cdot)$ are transformation operators, which are usually state transition matrixes; $\bigoplus$ is a operation, which is admissible to operate on two states; $f$ is the cost function or evaluation function.

As a intelligent optimization algorithm, the discrete state transition algorithm have the following five key elements:

(1) Representation of a solution. In discrete STA, we choose special representations, that is, the permutation of the set $\{1,2, \cdots, n\}$, which can be easily manipulated by some intelligent operators. The reason that we call the operators "intelligent" is due to their geometrical property (swap, shift, symmetry and substitute), and a intelligent operator has the same geometrical function for different representations. A big advantage of such representations and operators is that, after each state transformation, the newly created state is always 
feasible, avoiding the trouble into rounding off a continuous solution in other cases.

(2) Sampling in a candidate set. When a transformation operator is exerted on a current state, the next state is not deterministic, that is to say, there are possibly different choices for the next state. It is not difficult to imagine that all possible choices will constitute a candidate set, or a "neighborhood". Then we execute several times of transformation (called search enforcement $S E$ ) on current state, to sampling in the "neighborhood". Sampling is a very important factor in state transition algorithm, which can reduce the search space and avoid enumeration.

(3) Local exploitation and global exploration. In optimization algorithms, it is quite significant to design good local and global operators. The local exploitation can guarantee high precision of a solution and convergent performance of a algorithm, and the global exploration can avoid getting trapped into local minima or prevent premature convergence. In discrete optimization, it is extremely difficult to define a "good" local optimal solution due to its dependence on a problem's structure, which leads to the same difficulty in the definition of local exploitation and global exploration. Anyway, in the discrete state transition algorithm, we define the slow change to current solution by a transformation as local exploitation, while the big change to current solution by a transformation as global exploration.

(4) Self learning and regular communication. State transition algorithm behaves in two styles, one is individual-based, the other is population-based, which is certainly a extended version. The individual-based state transition algorithm focuses on self learning, in other words, with emphasis on the operators' designing and dynamic adjustment (details given in the following). Undoubtedly, communication among different states is a promising strategy for state transition algorithm, as indicated in [22]. Through communication, states can share information and cooperate with each other. However, how to communicate and when to communicate are key issues. In continuous state transition algorithm, intermittent exchange strategy was proposed, which means that states communicate with each other at a certain frequency in a regular way.

(5) Dynamic adjustment. It is a potentially useful strategy for state transition algorithm. In the iteration process of an intelligent algorithm, the fitness value can decrease sharply in the early stage, but it stagnates in the late stage, due to the static environment. As a result, some perturbation should be added to activate the environment. In fact, dynamic adjustment can be understood and implemented in various ways. For example, the alternative use of different local and global operators is dynamic adjustment to some extent. Then, we can change the search enforcement, vary the cost function, reduce the dimension, etc. Of course, "risk a bad solution in probability" is another dynamic adjustment, which is widely used in simulated annealing (SA). In SA, the Metropolis criterion [24] is used to accept a bad solution:

$$
\text { probability } p=\exp \left(\frac{-\triangle E}{k_{B} T}\right)
$$

where, $\Delta E=f\left(x_{k+1}\right)-f\left(x_{k}\right), k_{B}$ is the Boltzmann probability factor, $T$ is the temperature to regulate the process of annealing. In the early stage, temperature is high, and it has big probability to accept a bad solution, while in the late stage, temperature is low, and it has very small probability to accept a bad solution, which is the key point to guarantee the convergence. We can see that the Metropolis criterion has the ability to escape from local optimality, but on the other hand, it will miss some "good solutions" as well.

In this study, we focus on the individual-based STA, and the main process of discrete STA is shown in the pseudocode as follows

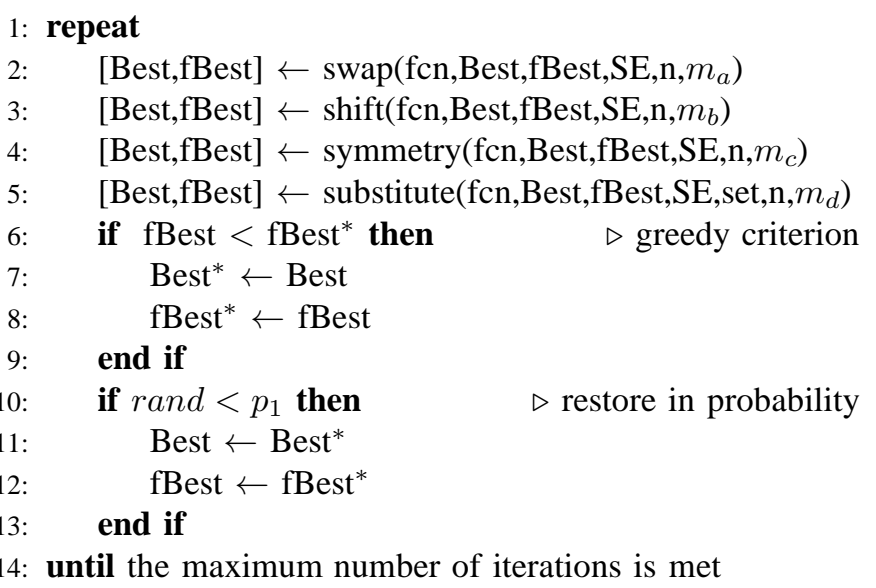

As for detailed explanations, swap function in above pseudocode is given as follows for example

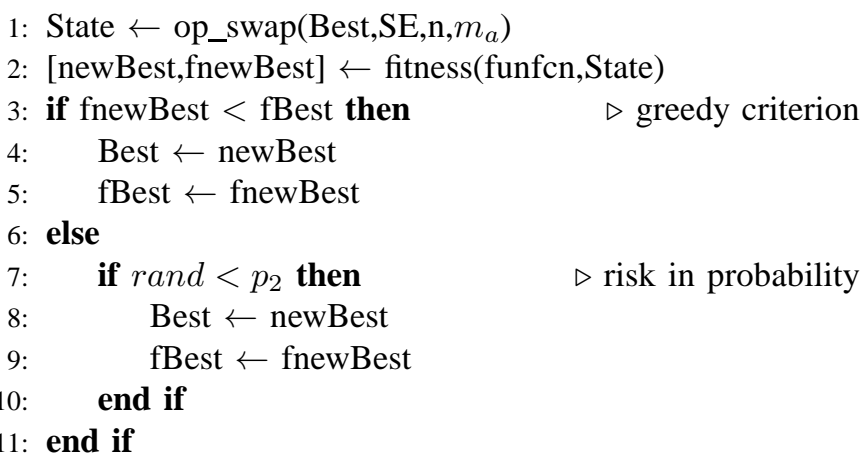

From the pseudocodes, we can find that in discrete STA, in the whole, "greedy criterion" is adopted to keep the incumbent "Best", , in the partial, a bad solution "Best" is accepted in each inner state transformation at a probability $p_{2}$, and in the same while, the "Best"" is restored in the outer iterative process at another probability $p_{1}$. The "risk a bad solution in probability" strategy aims to escape from local optimal, while the "greedy criterion" and "restore the incumbent best solution in probability" are to guarantee a good convergence.

\section{B. The representation, local and global operators}

In discrete STA, we use the index of the a commercial size as a representation for a solution to the optimal design problem. For example, if there are 8 pipes and for each pipe there are 3 choices, then the details of four special geometric operators are defined as follows 
(1) Swap transformation

$$
x_{k+1}=A_{k}^{s w a p}\left(m_{a}\right) x_{k},
$$

where, $A_{k}^{\text {swap }} \in \mathcal{Z}^{n \times n}$ is called swap permutation matrix, $m_{a}$ is a constant integer called swap factor to control the maximum number of positions to be exchanged, while the positions are random. If $m_{a}=2$, we call the swap operator local exploitation, and if $m_{a} \geq 3$, the swap operator is regarded as global exploration. Fig. 1 gives the function of the swap transformation graphically when $m_{a}=2$.

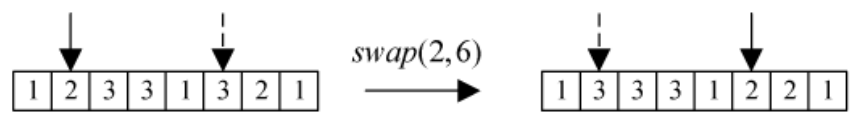

Fig. 1. illustration of the swap transformation

(2) Shift transformation

$$
x_{k+1}=A_{k}^{\text {shift }}\left(m_{b}\right) x_{k},
$$

where, $A_{k}^{\text {shift }} \in \mathcal{Z}^{n \times n}$ is called shift permutation matrix, $m_{b}$ is a constant integer called shift factor to control the maximum length of consecutive positions to be shifted. By the way, the selected position to be shifted after and positions to be shifted are chosen randomly. Similarly, shift transformation is called local exploitation and global exploration when $m_{b}=1$ and $m_{b} \geq 2$ respectively. To make it more clearly, if $m_{b}=1$, we set position 2 to be shifted after position 6 , as described in Fig. 2.

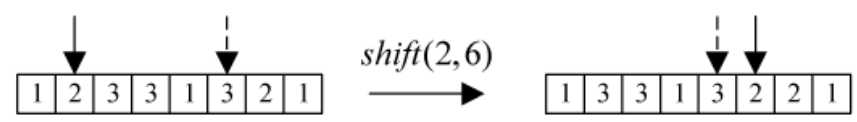

Fig. 2. illustration of the shift transformation

(3) Symmetry transformation

$$
x_{k+1}=A_{k}^{s y m}\left(m_{c}\right) x_{k},
$$

where, $A_{k}^{s y m} \in \mathcal{Z}^{n \times n}$ is called symmetry permutation matrix, $m_{c}$ is a constant integer called symmetry factor to control the maximum length of subsequent positions as center. By the way, the component before the subsequent positions and consecutive positions to be symmetrized are both created randomly. Considering that the symmetry transformation can make big change to current solution, it is intrinsically called global exploration. For instance, if $m_{c}=0$, let choose the position 3 , then the subsequent position or the center is $\{\emptyset\}$, the consecutive positions $\{4,5\}$ with components $(3,1)$, and the function of symmetry transformation is given in Fig. 3 .

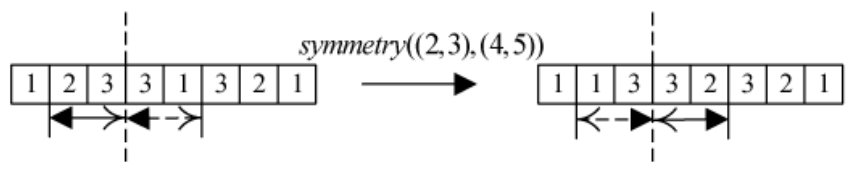

Fig. 3. illustration of the symmetry transformation

(4) Substitute transformation

$$
x_{k+1}=A_{k}^{s u b}\left(m_{d}\right) x_{k},
$$

where, $A_{k}^{s u b} \in \mathcal{Z}^{n \times n}$ is called substitute permutation matrix, $m_{d}$ is a constant integer called substitute factor to control the maximum number of positions to be substituted. By the way, the positions are randomly created. If $m_{d}=1$, we call the substitute operator local exploitation, and if $m_{d} \geq 2$, the substitute operator is regarded as global exploration. Fig. 4 gives the function of the substitute transformation vividly when $m_{d}=1$.

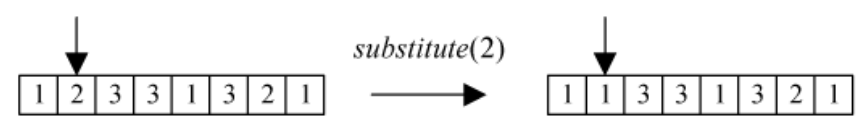

Fig. 4. illustration of the substitute transformation

\section{Theoretical analysis}

We give the definition of a global minimum for integer optimization as follows

$$
f\left(x^{*}\right) \leq f(x), \exists x^{*} \in \mathcal{X}, \forall x \in \mathcal{X},
$$

where, $\mathcal{X} \subset \mathcal{Z}^{n}$ is the feasible space. If (13) is satisfied, we say that $x^{*}$ is a global minimizer.

To give the convergence performance of the proposed discrete STA, we should introduce the general form of random search methods described by

1: Select a starting point $x_{0} \in \mathcal{X}$, and set $k \leftarrow 0$

2: repeat

3: $\quad$ Generate a candidate solution $x_{k}^{\prime} \in N\left(x_{k}\right) \cap \mathcal{X}$

4: $\quad$ if $f\left(x_{k}^{\prime}\right)<f\left(x_{k}\right)$ then

5: $\quad x_{k+1} \leftarrow x_{k}^{\prime}$

6: $\quad$ else

7: $\quad x_{k+1} \leftarrow x_{k}$

8: $\quad$ end if

9: $\quad k \leftarrow k+1$

10: until the specified termination criterion is met

where, the set $N\left(x_{k}\right)$ consists of the neighbors of the point $x_{k}$.

It was proved that the above general random search methods can converge in probability to an optimal solution, and it was also demonstrated that, to guarantee the convergence of an optimization algorithm, the criterion to accept a new solution is a key point [25]-[27]. The "greedy criterion" (always accept a better new solution) is sufficient to guarantee the convergence; nevertheless, accepting a relative worse solution as suggested in simulated annealing can also achieve asymptotic convergence [28]. In particular, random search methods differ in the choice of the neighborhood structure $N\left(x_{k}\right)$, and they influence the rate of convergence.

Theorem 1: The sequence generated by discrete STA can converge to a global minimizer in probability.

Proof: On the one hand, the discrete STA is a special case of the random search methods, since the "greedy criterion" is used as an external archive to keep the incumbent best solution, which can guarantee convergence of the proposed algorithms.

On the other hand, we have to show that the algorithm can capture a global minimizer probabilistically. Let suppose 
$x^{*}=\left(a_{1}, \cdots, a_{n}\right)$ is a global minimum solution, and $x_{N}=$ $\left(b_{1}, \cdots, b_{n}\right)$ is the $N$ th best solution. If $x_{N}=x^{*}$, according to the "greedy criterion", $f\left(x_{k}\right)=f\left(x_{N}\right), \forall k>N$, which means that it converges to $x^{*}$. Otherwise, there must exist a transformation, either swap, shift, symmetry or substitute, such that $x_{N+l_{1}}=\left(a_{1}, \cdots, b_{n}\right)$, which means that after $l_{1}$ iterations, $b_{1}$ will be changed into $a_{1}$. If $f\left(x_{N+l_{1}}\right)<f\left(x_{N}\right)$, the $x_{N+l_{1}}$ is kept as incumbent best for next iteration, else, $x_{N+l_{1}}$ is also kept as incumbent best for next iteration in probability. Following the similar way, after at most $l_{2}+\cdots+l_{n}$ iterations, $x_{N+l_{1}+\cdots+l_{n}}$ will be $\left(a_{1}, \cdots, a_{n}\right)$ in probability.

\section{Parameter selection}

In the state transformations, there are four factors to control the intensity between local search and global search. For simplicity and efficiency, the swap, shift and substitute operators are taken as local search, and the symmetry operator is considered as global search; therefore, we consistently make $m_{a}=2, m_{b}=1, m_{d}=1$ and $m_{c}=0$.

On the other hand, the restore probability $p_{1}$ and the risk probability $p_{2}$ play a significant role in the discrete STA, as described by the above theorems. To view the importance of the parameters, we arrange a Monte Carlo simulation study.

Considering the following optimization problem

$$
\min f^{*}
$$

where, $f^{*}$ is created by

$$
\begin{array}{ll}
\text { 1: } & \text { Initialize } f^{*} \leftarrow 0.5, f \leftarrow f^{*} \\
\text { 2: } & \text { repeat } \\
\text { 3: } & \text { if } f<r_{1} \text { then } \\
\text { 4: } & f \leftarrow r_{1} \\
\text { 5: } & \text { else if } r_{2}<p_{2} \text { then } \\
\text { 6: } & f \leftarrow r_{1} \\
\text { 7: } & \text { end if } \\
\text { 8: } & \text { if } f^{*}<f \text { then } \\
\text { 9: } & f^{*} \leftarrow f \\
0: & \text { end if } \\
1: & \text { if } r_{3}<p_{1} \text { then } \\
\text { 2: } & f \leftarrow f^{*} \\
\text { 3: } & \text { end if }
\end{array}
$$

$\triangleright$ risk in probability

$\triangleright$ greedy criterion

14: until the maximum number of iterations is met

here, $r_{1}, r_{2}, r_{3}$ are uniformly random numbers in $(0,1)$.

We test various groups of $\left(p_{1}, p_{2}\right)$ for the experiment, in which, the maximum number of iterations is $1 e 3$, and $1 e 4$ runs are carried out for each group. The experimental results are shown in Table I. Without loss of generality, the group $\left(p_{1}, p_{2}\right)=(0.1,0.1)$ is adopted in this paper for the following study due to its good performance and simplicity.

\section{IMPLEMENTATION OF THE DISCRETE STA}

The above discrete STA are essentially for unconstrained discrete optimization problem. To realize the optimal design of water distribution networks, we have to deal with some constraints. For the equality constraints on continuity of flow and conservation of energy, there exist some hydraulic analysis software packages such as EPANET [29], KYPIPE [30], in which the continuity and energy constraints are automatically satisfied. Considering that the continuity equations are linear, we can first fix some of pipe flows as known to solve the linear equations and then substitute them into the energy equations, which can reduce the computational complexity of solving continuity equations (linear) and energy equations (nonlinear) simultaneously. It is not difficult to imagine that the number of nonlinear equations equals to that of the simple closed loops or required independent paths in a network, and then a NewtonRaphson method is used to solve the nonlinear equations.

For the minimum pressure head constraints, the most commonly used technique is the penalty function method, adding a penalty term when the corresponding constraint is violated. For example, the following scheme

$$
f_{\text {penal }}=p c \sum_{i=1}^{N P} \max \left\{0, H_{i \min }-H_{i}\right\}^{\rho}
$$

where, $p c$ is the penalty coefficient, and $\rho$ is normally 1 or 2 ( $\rho=1$ in this study). Finally, the total cost is

$$
f_{\text {cost }}=f_{\text {obj }}+f_{\text {penal }} \text {. }
$$

A brief description of the steps using discrete STA is given in the following

1) Creat initial Best solution. Generate a group of candidate solutions randomly (the size is the search enforcement, $S E$ ) and then select the fittest solution. Let Best ${ }^{*}=$ Best and store Best* .

2) Update the Best. Use swap transformation to generate a group of candidate solutions on the basis of Best. If the fittest of the candidate solutions is better than Best, then accept the fittest solution as Best; otherwise, accept the fittest solution as Best in a probability $p_{2}$. Similar procedures are adaptive to shift, symmetry and substitute transformations.

3) Update the Best*. The Best* is updating only when Best is better than Best*.

4) Restore the Best. The Best is restored to Best* in a probability $p_{1}$.

5) Go back to repeat step 2 until the stopping criterion is met.

Remark 1: We should notice that once a solution is given, then the flow in each pipe is determined by solving the nonlinear equations, and then we can evaluate whether the minimum pressure head is satisfied and decide the corresponding penalty term to each head pressure constraint.

\section{CAse STUdies}

We investigate the performance of the proposed discrete STA by three well-known water distribution networks, namely, the Two-Loop network, the Hanoi network and the New York network. We first give a detailed study of the TwoLoop problem to show that the network system with eight unknowns governed by six linear equations and two nonlinear equations can be reduced to only two unknowns governed by two nonlinear equations. This Two-Loop case is also fully trained to study the effect of penalty coefficient and search enforcement on the performance of the algorithm. Based on the experience gained from the case, the known best solutions for the other two networks are also achieved by the algorithm. 
TABLE I

A Monte Carlo simulation Study

\begin{tabular}{cccccc}
\hline$\left(p_{1} \backslash p_{2}\right)$ & 0.1 & 0.3 & 0.5 & 0.7 & 0.9 \\
\hline 0.1 & $\mathbf{9 . 9 2 5 4 e - 4} \pm \mathbf{9 . 8 2 7 4 e - 4}{ }^{1}$ & $0.0010 \pm 9.8255 \mathrm{e}-4$ & $0.0010 \pm 0.0010$ & $\mathbf{9 . 9 0 2 7 e - 4} \pm \mathbf{9 . 9 4 9 8 e - 4}$ & $\mathbf{9 . 7 4 2 3 e - 4} \pm \mathbf{9 . 8 1 6 3 e - 4}$ \\
0.3 & $0.0010 \pm 9.9359 \mathrm{e}-4$ & $0.0010 \pm 0.0010$ & $0.0010 \pm 0.0010$ & $\mathbf{9 . 8 7 3 6 e - 4} \pm \mathbf{9 . 8 1 5 9 e - 4}$ & $9.9254 \mathrm{e}-4 \pm 0.0010$ \\
0.5 & $0.0010 \pm 9.9755 \mathrm{e}-4$ & $\mathbf{9 . 9 2 4 9 e - 4} \pm \mathbf{9 . 7 5 4 7 e - 4}$ & $0.0010 \pm 0.0010$ & $0.0010 \pm 0.0010$ & $0.0010 \pm 0.0010$ \\
0.7 & $0.0010 \pm 0.0010$ & $0.0010 \pm 0.0010$ & $0.0010 \pm 0.0010$ & $\mathbf{9 . 9 4 5 6 e - 4} \pm \mathbf{9 . 8 7 3 0 e - 4}$ & $\mathbf{9 . 9 6 0 9 e - 4} \pm \mathbf{9 . 9 9 9 1 e - 4}$ \\
0.9 & $0.0010 \pm 9.9637 \mathrm{e}-4$ & $0.0010 \pm 9.9667 \mathrm{e}-4$ & $\mathbf{9 . 8 2 5 8 e - 4} \pm \mathbf{9 . 8 2 6 1 e - 4}$ & $0.0010 \pm 0.0010$ & $0.0010 \pm 0.0010$ \\
\hline
\end{tabular}

1 indicates mean \pm standard deviation

\section{A. two-loop network}

The layout of the two-loop network is given in Fig. 5 There are a single reservoir at a $210-\mathrm{m}$ fixed head and eight pipes all with 1000-m long. The node data and cost data are given in Table 【 and Table II and the minimum acceptable pressure requirements are all $30-\mathrm{m}$ above the ground level. The HazenWilliams coefficient $C$ is assumed to be 130 for the two-loop network.

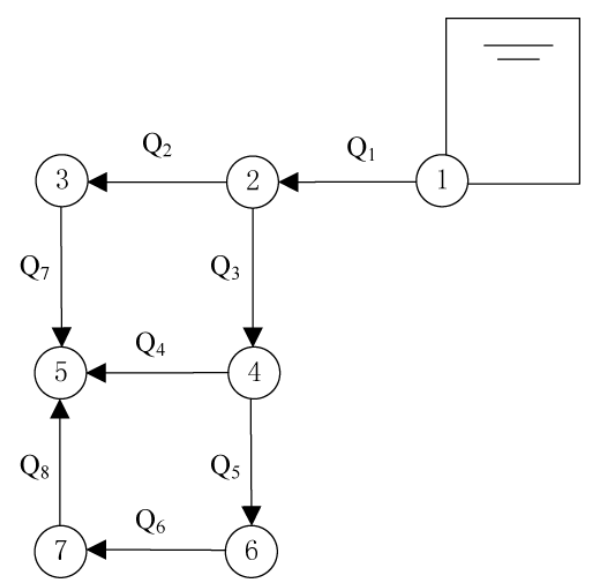

Fig. 5. two loop network

TABLE II

NODE DATA FOR THE TWO LOOP NETWORK

\begin{tabular}{ccc}
\hline Node & Demand $\left(m^{3} / h\right)$ & Ground level $(m)$ \\
\hline 1 & -1120.0 & 210.00 \\
2 & 100.0 & 150.00 \\
3 & 100.0 & 160.00 \\
4 & 120.0 & 155.00 \\
5 & 270.0 & 150.00 \\
6 & 330.0 & 165.00 \\
7 & 200.0 & 160.00 \\
\hline
\end{tabular}

In this case study, we give an illustrative procedure of how to reduce the complexity of solving the linear and nonlinear equations. The flow continuity equations of the two-loop
TABLE III

COST DATA FOR THE TWO LOOP NETWORK

\begin{tabular}{cccccc}
\hline No. & Diameter (in.) & Cost $(\$ / \mathrm{m})$ & No. & Diameter (in.) & Cost $(\$ / \mathrm{m})$ \\
\hline 1 & 1 & 2 & 8 & 12 & 50 \\
2 & 2 & 5 & 9 & 14 & 60 \\
3 & 3 & 8 & 10 & 16 & 90 \\
4 & 4 & 11 & 11 & 18 & 130 \\
5 & 6 & 16 & 12 & 20 & 170 \\
6 & 8 & 23 & 13 & 22 & 300 \\
7 & 10 & 32 & 14 & 24 & 550 \\
\hline
\end{tabular}

${ }^{2} 1$ in. $=2.54 \mathrm{~cm}$

network are given as follows

$$
\left\{\begin{array}{l}
-Q_{1}+Q_{2}+Q_{3}+D M_{2}=0 \\
-Q_{2}+Q_{7}+D M_{3}=0 \\
-Q_{3}+Q_{4}+Q_{5}+D M_{4}=0 \\
-Q_{7}-Q_{8}-Q_{4}+D M_{5}=0 \\
-Q_{5}+Q_{6}+D M_{6}=0 \\
-Q_{6}+Q_{8}+D M_{7}=0
\end{array}\right.
$$

Let $Q_{4}, Q_{6}$ be fixed, then

$$
\left\{\begin{array}{l}
Q_{1}=D M_{2}+D M_{3}+D M_{4}+D M_{5}+D M_{6}+D M_{7} \\
Q_{2}=D M_{3}+D M_{5}+D M_{7}-Q_{4}-Q_{6} \\
Q_{3}=D M_{4}+D M_{6}+Q_{4}+Q_{6} \\
Q_{5}=D M_{6}+Q_{6} \\
Q_{7}=D M_{5}+D M_{7}-Q_{4}-Q_{6} \\
Q_{8}=Q_{6}-D M_{7}
\end{array}\right.
$$

The energy conservation equations can be formulated as

$$
\left\{\begin{array}{l}
r_{3} Q_{3}\left|Q_{3}\right|^{\alpha-1}+r_{4} Q_{4}\left|Q_{4}\right|^{\alpha-1}-r_{7} Q_{7}\left|Q_{7}\right|^{\alpha-1}-r_{2} Q_{2}\left|Q_{2}\right|^{\alpha-1}=0 \\
r_{5} Q_{5}\left|Q_{5}\right|^{\alpha-1}+r_{6} Q_{6}\left|Q_{6}\right|^{\alpha-1}+r_{8} Q_{8}\left|Q_{8}\right|^{\alpha-1}-r_{4} Q_{4}\left|Q_{4}\right|^{\alpha-1}=0
\end{array}\right.
$$

and the head loss equations

$$
\left\{\begin{array}{l}
H_{2}=H e a d-r_{1} Q_{1}\left|Q_{1}\right|^{\alpha-1}-G_{2} \geq H_{2 \min } \\
H_{3}=H_{2}-r_{2} Q_{2}\left|Q_{2}\right|^{\alpha-1}-G_{3} \geq H_{3 \min } \\
H_{4}=H_{2}-r_{3} Q_{3}\left|Q_{3}\right|^{\alpha-1}-G_{4} \geq H_{4 \min } \\
H_{5}=H_{4}-r_{4} Q_{4}\left|Q_{4}\right|^{\alpha-1}-G_{5} \geq H_{5 \min } \\
H_{6}=H_{4}-r_{5} Q_{5}\left|Q_{5}\right|^{\alpha-1}-G_{6} \geq H_{6 \text { min }} \\
H_{7}=H_{6}-r_{6} Q_{6}\left|Q_{6}\right|^{\alpha-1}-G_{7} \geq H_{7 \min }
\end{array}\right.
$$

where, $G_{i}(i=2, \cdots, 7)$ is the ground level.

Remark 2: It should be noted that we only need to solve the nonlinear system (19) with two unknowns $\left(Q_{4}, Q_{6}\right)$. 
For the Two-Loop network, we have to select a diameter to each pipe, and for each pipe there are 14 choices. It is not difficult to imagine that when choosing a numerical order (No.), it corresponds to an exact diameter. That is the reason why the discrete STA use the permutation of $\{1,2, \cdots, n\}$ as its decision variables and all the intelligent operators are operated on a certain permutation.

Next, we conduct a eremitical study of the the two loop network by the proposed discrete STA to investigate the influence of the remained parameters, namely, the search enforcement $(S E)$ and the penalty coefficient $(p c)$. We set $S E$ to be $0.5,1,2,3$ and 4 times of the dimension of decision variable. Considering that the average cost times the average pipe length is $1.0335 \mathrm{e} 5$ and the average of the minimum pressure heads is 30 , the order of magnitude for $p c$ is set at $1 \mathrm{e} 4$. On this situation, $p c$ is fixed at $1 e 4,2 e 4,4 e 4,8 e 4$ and $1 e 5$, or increases from $1 e 4$ to $1 e 5$ in a linear way. The maximum number of iterations is set at $2 e 2$, and a total of 20 runs are executed for each group of search enforcement $S E$ and penalty coefficient $p c$.

As can be seen from Table IV for a fixed $S E$, the search ability is declining as the $p c$ increases, but the feasibility rate increases simultaneously with the $p c$. For a fixed $p c$, the search ability is increasing as the $S E$ increases from 4 to 8 but declining as the $S E$ increases any more. When the $p c$ varies in the iterative process, the performance is not the best but much more satisfactory than a constant one to some extent. By observation, we can find that setting $S E$ to be the dimensionality of the decision variable is a good choice, and in this setting environment, $p c=2 e 4$ is a good penalty coefficient. Fig. 6 gives the iterative curves of the gained best solutions when $S E=8$ and $p c=2 e 4$, respectively. It should be emphasized that best solutions are all 419,000.

Remark 3: Under the circumstance, the minimum function evaluations to achieve the best known solution is 2048, which takes up $0.0001387 \%$ of all possible combinations $\left(14^{8}=\right.$ $1.4758 e 9)$.

Table $\mathrm{V}$ gives the best solutions gained by various algorithms, and it can be found that STA can achieve the best known solution in this case. It should be noted that the same solution was also achieved by GA [9], SA [11] and HS [15] with function evaluations at 250,000, 70, 000 and 5,000, respectively. Although the solution in [6] is even better, it should be noted that it brings pipe segments. The pressure heads for the Two-Loop network obtained by various algorithms are given in Table VI.

\section{B. Hanoi network}

The layout of the Hanoi network is given in Fig. 7 There are 32 nodes, 34 pipes and 3 loops in this network system. At node 1 , there exists a reservoir with a $100-\mathrm{m}$ fixed head. The cost data, and pipe and node data are given in Table VII and Table VIII respectively. The minimum acceptable pressure requirements at all nodes are also fixed at $30 \mathrm{~m}$ and the HazenWilliams coefficient $C$ is assumed to be 130 as well.

From the experience gained from the training of the TwoLoop network, the search enforcement $S E$ does not affect the
TABLE VII

COST DATA FOR THE HANOI NETWORK

\begin{tabular}{ccc}
\hline No. & Diameter (in.) & Cost $(\$ / \mathrm{m})$ \\
\hline 1 & 12 & 45.726 \\
2 & 16 & 70.400 \\
3 & 20 & 98.387 \\
4 & 24 & 129.333 \\
5 & 30 & 180.748 \\
6 & 40 & 278.280 \\
\hline
\end{tabular}

performance of the discrete STA explicitly, but the penalty coefficient $p c$ plays a significant role in the search ability and the solution feasibility, and a good penalty coefficient can be evaluated from the order of magnitude the same as the average pipe length times the minimum pressure heads.

For the Hanoi network, the search enforcement $S E$ is set at 20 , and the penalty coefficient $p c$ is fixed at $4 e 4$, or varies from $4 e 4$ to $1 e 5$ in a linearly increasing way. The maximum number of iterations is set at $1 e 3$, and a total of 20 runs are executed for both fixed and variable $p c$. Fig. 8 gives the iterative curves of best solutions and changes in 20 runs for the Hanoi problem with fixed and variable $p c$ respectively. It is shown that the best solution is hit only once by the STA with fixed $p c$.

Remark 4: Under the circumstance, the minimum function evaluations to achieve the best known solution is 23,240, which takes up $8.1114 e-21 \%$ of all possible combinations $\left(6^{34}=2.8651 e 26\right)$.

Table IX gives the best solutions gained by various algorithms, and it can be found that STA with fixed $p c$ can achieve the best known solution in this case at the cost of 6.056 million dollars, while the solution of STA with variable $p c$ get a solution at the cost of 6.065 million dollars. Savic and Walters [9] used the GA to obtain the solution with 1,000,000 function evaluations. The solution gained by Zecchin et al. [14] using ACO need 100,000 function evaluations. The exactly same solution was achieved by SA [11] and HS [15] as well, with the function evaluations at 53,000 and 200,000, respectively. The pressure heads for the Hanoi network obtained by various algorithms are given in Table $\mathrm{X}$

\section{New York network}

The layout of the New York network is given in Fig. 9 There are 20 nodes, 21 pipes and 1 loop in this network system. At node 1 , there exists a reservoir with $300-\mathrm{ft}$ fixed head. The New York problem is different from other two cases, because there already exist pipes in the old system. The common objective of this problem is to determine additional parallel pipes added to the existing ones to meet increased water demands while maintaining the minimum pressure requirements. The the cost data, pipe and node data are given in Table XI and Table XII respectively. The Hazen-Williams coefficient $C$ is assumed to be 100 in this case.

For the New York network, the search enforcement $S E$ is also set at 10 , and the penalty coefficient $p c$ is fixed at $2 e 6$, or varies from $1 e 6$ to $1 e 7$ in a linearly increasing way. The maximum number of iterations is set at $2 e 3$, and a total of 
TABLE IV

A EMPIRICAL STUDY OF THE TWO LOOP NETWORK

\begin{tabular}{|c|c|c|c|c|c|c|}
\hline$(S E \backslash p c)$ & $1 \mathrm{e} 4$ & $2 \mathrm{e} 4$ & $4 \mathrm{e} 4$ & $8 \mathrm{e} 4$ & $1 \mathrm{e} 5$ & $1 \mathrm{e} 4 \rightarrow 1 \mathrm{e} 5$ \\
\hline 4 & $\begin{array}{l}4.2978 \mathrm{e} 5 \pm 1.4882 \mathrm{e} 4 \\
(55 \%)^{3}\end{array}$ & $\begin{array}{l}4.3631 \mathrm{e} 5 \pm 1.3394 \mathrm{e} 4 \\
(85 \%)\end{array}$ & $\begin{array}{l}4.5184 \mathrm{e} 5 \pm 2.3575 \mathrm{e} 4 \\
(95 \%)\end{array}$ & $\begin{array}{l}4.5063 \mathrm{e} 5 \pm 1.7400 \mathrm{e} 4 \\
(95 \%)\end{array}$ & $\begin{array}{l}4.4190 \mathrm{e} 5 \pm 1.6121 \mathrm{e} 4 \\
(95 \%)\end{array}$ & $\begin{array}{l}4.4368 \mathrm{e} 5 \pm 1.8563 \mathrm{e} 4 \\
(90 \%)\end{array}$ \\
\hline 8 & $\begin{array}{l}4.2195 \mathrm{e} 5 \pm 1.4853 \mathrm{e} 4 \\
(65 \%)\end{array}$ & $\begin{array}{l}4.3181 \mathrm{e} 5 \pm 1.3870 \mathrm{e} 4 \\
(85 \%)\end{array}$ & $\begin{array}{l}4.3526 \mathrm{e} 5 \pm 1.2721 \mathrm{e} 4 \\
(90 \%)\end{array}$ & $\begin{array}{l}4.3577 \mathrm{e} 5 \pm 1.2903 \mathrm{e} 4 \\
(95 \%)\end{array}$ & $\begin{array}{l}4.4085 \mathrm{e} 5 \pm 1.5853 \mathrm{e} 4 \\
(90 \%)\end{array}$ & $\begin{array}{l}4.3620 \mathrm{e} 5 \pm 1.5702 \mathrm{e} 4 \\
(80 \%)\end{array}$ \\
\hline 16 & $\begin{array}{l}4.2682 \mathrm{e} 5 \pm 1.2946 \mathrm{e} 4 \\
(75 \%)\end{array}$ & $\begin{array}{l}4.3340 \mathrm{e} 5 \pm 1.5347 \mathrm{e} 4 \\
(80 \%)\end{array}$ & $\begin{array}{l}4.3410 \mathrm{e} 5 \pm 1.2004 \mathrm{e} 4 \\
(90 \%)\end{array}$ & $\begin{array}{l}431550 \pm 1.4406 \mathrm{e} 4 \\
(100 \%)\end{array}$ & $\begin{array}{l}4.3458 \mathrm{e} 5 \pm 1.4992 \mathrm{e} 4 \\
(90 \%)\end{array}$ & $\begin{array}{l}433600 \pm 1.4207 \mathrm{e} 4 \\
(100 \%)\end{array}$ \\
\hline 24 & $\begin{array}{l}4.2380 \mathrm{e} 5 \pm 1.2756 \mathrm{e} 4 \\
(75 \%)\end{array}$ & $\begin{array}{l}4.3193 \mathrm{e} 5 \pm 1.2898 \mathrm{e} 4 \\
(95 \%)\end{array}$ & $\begin{array}{l}4.3555 \mathrm{e} 5 \pm 1.5049 \mathrm{e} 4 \\
(90 \%)\end{array}$ & $\begin{array}{l}440950 \pm 1.4417 \mathrm{e} 4 \\
(100 \%)\end{array}$ & $\begin{array}{l}432600 \pm 1.5398 \mathrm{e} 4 \\
(100 \%)\end{array}$ & $\begin{array}{l}4.3073 \mathrm{e} 5 \pm 1.3252 \mathrm{e} 4 \\
(95 \%)\end{array}$ \\
\hline 32 & $\begin{array}{l}4.2686 \mathrm{e} 5 \pm 1.5549 \mathrm{e} 4 \\
(55 \%)\end{array}$ & $\begin{array}{l}4.3046 \mathrm{e} 5 \pm 1.5523 \mathrm{e} 4 \\
(80 \%)\end{array}$ & $\begin{array}{l}4.3376 \mathrm{e} 5 \pm 1.3900 \mathrm{e} 4 \\
(95 \%)\end{array}$ & $\begin{array}{l}451400 \pm 5.4648 \mathrm{e} 4 \\
(100 \%)\end{array}$ & $\begin{array}{l}4.3600 \mathrm{e} 5 \pm 1.7731 \mathrm{e} 4 \\
(85 \%)\end{array}$ & $\begin{array}{l}4.3305 \mathrm{e} 5 \pm 1.4657 \mathrm{e} 4 \\
(95 \%)\end{array}$ \\
\hline
\end{tabular}

${ }^{3}$ indicates the percentage of feasible solutions
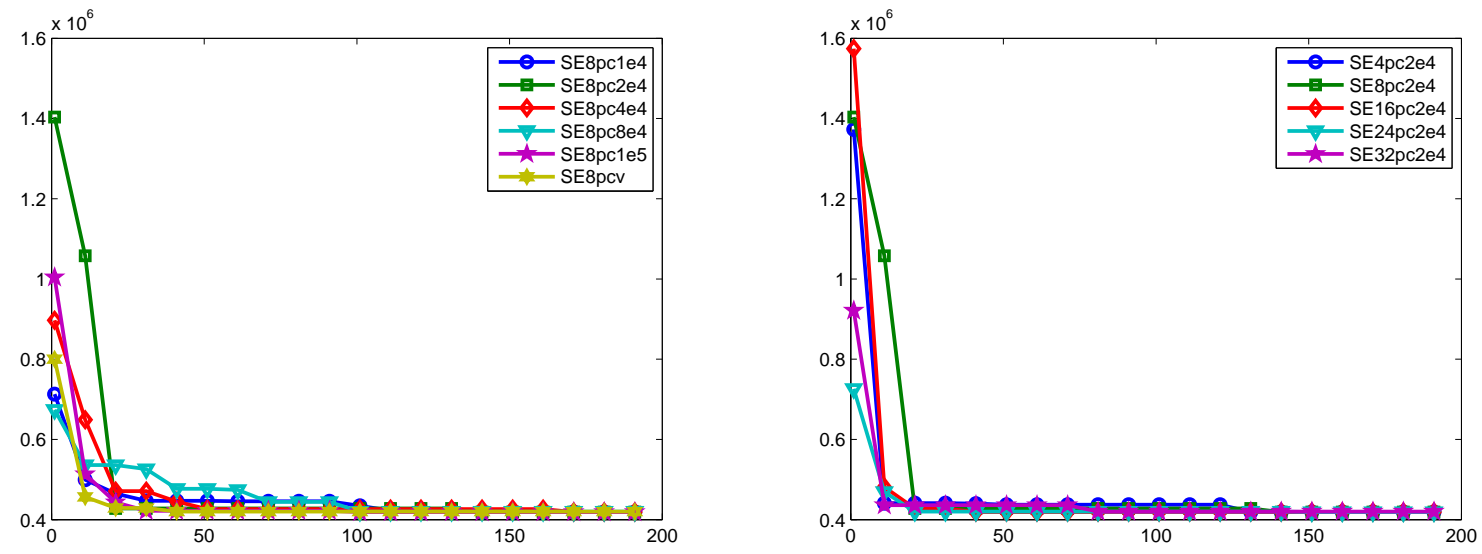

Fig. 6. Iterative curves of best solutions when $S E=8$ and $p c=2 e 4$ for the Two-Loop problem, respectively

TABLE V

SOLUTIONS FOR THE TWO LOOP NETWORK

\begin{tabular}{|c|c|c|c|c|c|}
\hline Pipe & Alperovits and Shamir [3] & Goulter et al. [5] & Kessler and Shamir [6] & STA (fixed) & STA (variable) \\
\hline \multirow[t]{2}{*}{1} & 20 & 20 & 18 & 18 & 18 \\
\hline & 18 & 18 & & & \\
\hline \multirow[t]{2}{*}{2} & 8 & 10 & 12 & 10 & 10 \\
\hline & 6 & & 10 & & \\
\hline 3 & 18 & 16 & 16 & 16 & 16 \\
\hline \multirow[t]{2}{*}{4} & 8 & 6 & 3 & 4 & 4 \\
\hline & 6 & 4 & 2 & & \\
\hline \multirow[t]{2}{*}{5} & 16 & 16 & 16 & 16 & 16 \\
\hline & & 14 & 14 & & \\
\hline \multirow[t]{2}{*}{6} & 12 & 12 & 12 & 10 & 10 \\
\hline & 10 & 10 & 10 & & \\
\hline \multirow[t]{2}{*}{7} & 6 & 10 & 10 & 10 & 10 \\
\hline & & 8 & 8 & & \\
\hline \multirow[t]{2}{*}{8} & 6 & 2 & 3 & 1 & 1 \\
\hline & 4 & 1 & 2 & & \\
\hline $\operatorname{Cost}(\$)$ & 497,525 & 435,015 & 417,500 & 419,000 & 419,000 \\
\hline
\end{tabular}


TABLE VI

PRESSURE HEADS FOR THE TWO-LOOP NETWORK

\begin{tabular}{ccccc}
\hline Node & Alperovits and Shamir [3] & Goulter et al. [5] & Kessler and Shamir [6] & STA (fixed and variable) \\
\hline 2 & 53.96 & 54.30 & 53.26 & 53.24 \\
3 & 32.32 & 33.19 & 30.08 & 30.49 \\
4 & 44.97 & 44.19 & 43.64 & 43.44 \\
5 & 32.31 & 32.32 & 30.10 & 33.78 \\
6 & 31.19 & 31.19 & 30.08 & 30.43 \\
7 & 31.57 & 31.57 & 30.09 & 30.54 \\
\hline
\end{tabular}

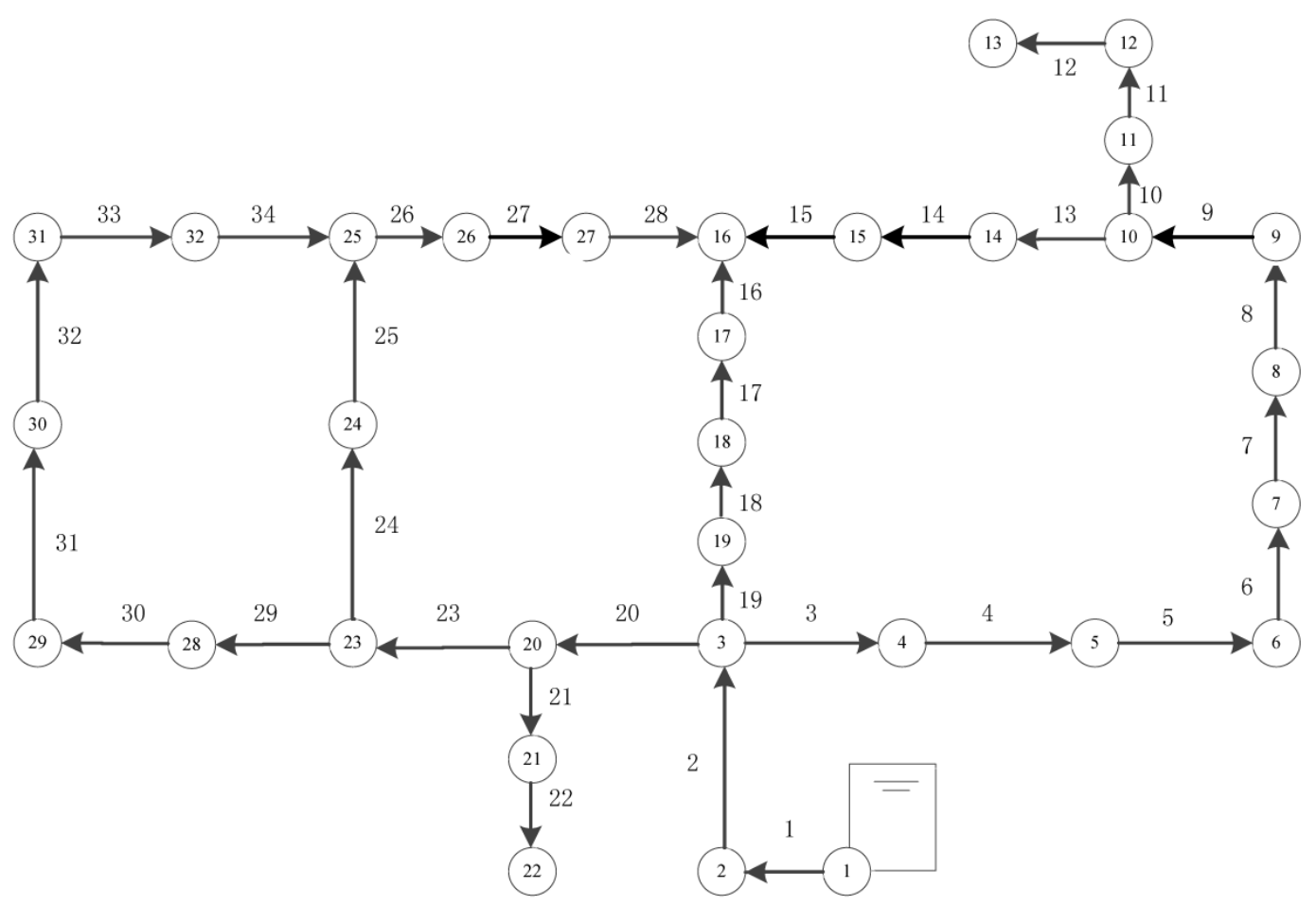

Fig. 7. The Hanoi network

TABLE VIII

PIPE AND NODE DATA FOR THE HANOI NETWORK

\begin{tabular}{cccccccc}
\hline Pipe & Length $(m)$ & Pipe & Length $(m)$ & Node & Demand $\left(m^{3} / h\right)$ & Node & Demand $\left(m^{3} / h\right)$ \\
\hline 1 & 100 & 18 & 800 & 1 & -19940 & 18 & 1345 \\
2 & 1350 & 19 & 400 & 2 & 890 & 19 & 60 \\
3 & 900 & 20 & 2200 & 3 & 850 & 20 & 1275 \\
4 & 1150 & 21 & 1500 & 4 & 130 & 21 & 930 \\
5 & 1450 & 22 & 500 & 5 & 725 & 22 & 485 \\
6 & 450 & 23 & 2650 & 6 & 1005 & 23 & 1045 \\
7 & 850 & 24 & 1230 & 7 & 1350 & 24 & 820 \\
8 & 850 & 25 & 1300 & 8 & 550 & 25 & 170 \\
9 & 800 & 26 & 850 & 9 & 525 & 26 & 900 \\
10 & 950 & 27 & 300 & 10 & 525 & 27 & 370 \\
11 & 1200 & 28 & 750 & 11 & 500 & 28 & 290 \\
12 & 3500 & 29 & 1500 & 12 & 560 & 29 & 360 \\
13 & 800 & 30 & 2000 & 13 & 940 & 30 & 360 \\
14 & 500 & 31 & 1600 & 14 & 615 & 31 & 105 \\
15 & 550 & 32 & 150 & 15 & 280 & 32 & 805 \\
16 & 2730 & 33 & 860 & 16 & 310 & - & - \\
17 & 1750 & 34 & 950 & 17 & 865 & - & - \\
\hline
\end{tabular}


TABLE IX

SOLUTIONS FOR THE HANOI NETWORK

\begin{tabular}{|c|c|c|c|c|c|c|c|}
\hline Pipe & $\begin{array}{l}\text { Savic and } \\
\text { Walters } 9\end{array}$ & $\begin{array}{l}\text { Zecchin } \\
\text { et al. 14 }\end{array}$ & $\begin{array}{l}\text { Haghighi } \\
\text { et al. [19] }\end{array}$ & $\begin{array}{l}\text { STA (fixed) } \\
\omega=10.6744\end{array}$ & $\omega=10.5088$ & $\begin{array}{l}\text { STA (variable) } \\
\omega=10.6744\end{array}$ & $\omega=10.5088$ \\
\hline 1 & 40 & 40 & 40 & 40 & 40 & 40 & 40 \\
\hline 2 & 40 & 40 & 40 & 40 & 40 & 40 & 40 \\
\hline 3 & 40 & 40 & 40 & 40 & 40 & 40 & 40 \\
\hline 4 & 40 & 40 & 40 & 40 & 40 & 40 & 40 \\
\hline 5 & 40 & 40 & 40 & 40 & 40 & 40 & 40 \\
\hline 6 & 40 & 40 & 40 & 40 & 40 & 40 & 40 \\
\hline 7 & 40 & 40 & 40 & 40 & 40 & 40 & 40 \\
\hline 8 & 40 & 40 & 40 & 40 & 40 & 40 & 40 \\
\hline 9 & 40 & 40 & 30 & 40 & 40 & 30 & 30 \\
\hline 10 & 30 & 30 & 30 & 30 & 30 & 30 & 30 \\
\hline 11 & 24 & 24 & 30 & 24 & 24 & 30 & 30 \\
\hline 12 & 24 & 24 & 24 & 24 & 24 & 24 & 24 \\
\hline 13 & 20 & 20 & 16 & 20 & 20 & 20 & 20 \\
\hline 14 & 16 & 12 & 12 & 16 & 16 & 12 & 16 \\
\hline 15 & 12 & 12 & 12 & 12 & 12 & 12 & 12 \\
\hline 16 & 12 & 12 & 16 & 12 & 12 & 12 & 12 \\
\hline 17 & 16 & 20 & 20 & 16 & 16 & 20 & 16 \\
\hline 18 & 20 & 24 & 24 & 24 & 20 & 20 & 24 \\
\hline 19 & 20 & 20 & 24 & 20 & 20 & 24 & 20 \\
\hline 20 & 40 & 40 & 40 & 40 & 40 & 40 & 40 \\
\hline 21 & 20 & 20 & 20 & 20 & 20 & 20 & 20 \\
\hline 22 & 12 & 12 & 12 & 12 & 12 & 12 & 12 \\
\hline 23 & 40 & 40 & 40 & 40 & 40 & 40 & 40 \\
\hline 24 & 30 & 30 & 30 & 30 & 30 & 30 & 30 \\
\hline 25 & 30 & 30 & 30 & 30 & 30 & 30 & 30 \\
\hline 26 & 20 & 20 & 20 & 20 & 20 & 20 & 20 \\
\hline 27 & 12 & 12 & 12 & 12 & 12 & 12 & 12 \\
\hline 28 & 12 & 12 & 12 & 12 & 12 & 12 & 12 \\
\hline 29 & 16 & 16 & 16 & 16 & 16 & 16 & 16 \\
\hline 30 & 16 & 16 & 12 & 16 & 12 & 16 & 12 \\
\hline 31 & 12 & 12 & 12 & 12 & 12 & 12 & 12 \\
\hline 32 & 12 & 12 & 16 & 12 & 16 & 16 & 16 \\
\hline 33 & 16 & 16 & 20 & 16 & 16 & 16 & 16 \\
\hline 34 & 20 & 20 & 24 & 20 & 24 & 20 & 24 \\
\hline Cost $(\$$ millions $)$ & 6.073 & 6.134 & 6.190 & 6.097 & 6.056 & 6.109 & 6.065 \\
\hline
\end{tabular}
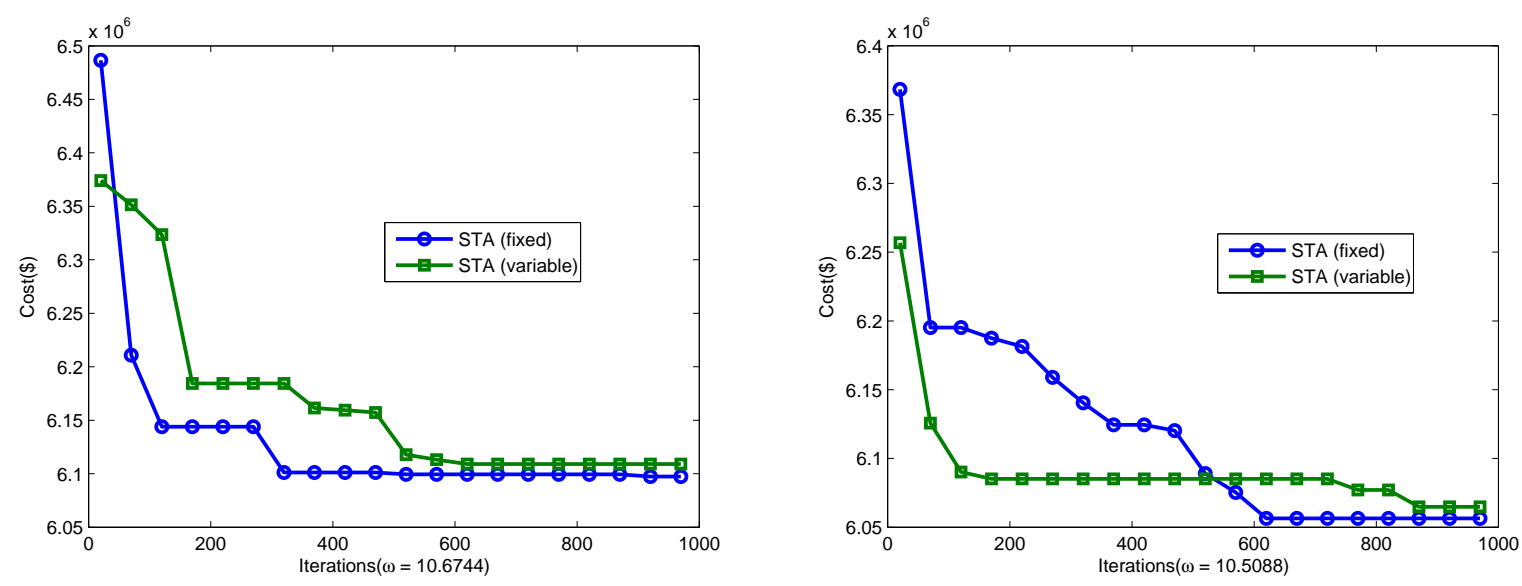

Fig. 8. Iterative curves of best solutions using STA for the Hanoi problem when $\omega$ is 10.6744 and 10.5088 , respectively 
TABLE X

PRESSURE HEADS FOR THE HANOI NETWORK

\begin{tabular}{|c|c|c|c|c|c|c|}
\hline Node & $\begin{array}{l}\text { Savic and } \\
\text { Walters [9] }\end{array}$ & $\begin{array}{l}\text { Haghighi } \\
\text { et al. [19] }\end{array}$ & $\begin{array}{l}\text { STA } \quad \text { (fixed) } \\
\omega=10.6744\end{array}$ & $\omega=10.5088$ & $\begin{array}{l}\text { STA (variable) } \\
\omega=10.6744\end{array}$ & $\omega=10.5088$ \\
\hline 1 & 100.00 & 100.00 & 100.00 & 100.00 & 100.00 & 100.00 \\
\hline 2 & 97.16 & 97.08 & 97.14 & 97.17 & 97.14 & 97.17 \\
\hline 3 & 61.95 & 60.82 & 61.64 & 61.99 & 61.64 & 61.99 \\
\hline 4 & 57.21 & 56.38 & 56.90 & 57.23 & 57.08 & 57.34 \\
\hline 5 & 51.33 & 50.88 & 51.02 & 51.31 & 51.42 & 51.56 \\
\hline 6 & 45.13 & 45.13 & 44.82 & 45.07 & 45.49 & 45.48 \\
\hline 7 & 43.68 & 43.81 & 43.36 & 43.61 & 44.10 & 44.06 \\
\hline 8 & 41.93 & 42.28 & 41.63 & 41.85 & 42.48 & 42.37 \\
\hline 9 & 40.54 & 41.09 & 40.25 & 40.44 & 41.20 & 41.02 \\
\hline 10 & 40.34 & 37.61 & 39.23 & 39.40 & 37.39 & 37.01 \\
\hline 11 & 38.79 & 36.01 & 37.67 & 37.85 & 35.83 & 35.45 \\
\hline 12 & 38.78 & 34.83 & 34.24 & 34.43 & 34.68 & 34.30 \\
\hline 13 & 34.58 & 30.53 & 30.03 & 30.24 & 30.46 & 30.10 \\
\hline 14 & 36.59 & 32.06 & 35.61 & 35.49 & 34.64 & 33.66 \\
\hline 15 & 34.71 & 30.96 & 33.87 & 33.44 & 30.86 & 32.17 \\
\hline 16 & 32.08 & 31.13 & 31.61 & 30.36 & 30.38 & 30.53 \\
\hline 17 & 33.36 & 39.28 & 33.56 & 30.51 & 38.00 & 33.20 \\
\hline 18 & 43.32 & 50.04 & 49.94 & 44.29 & 44.89 & 50.16 \\
\hline 19 & 55.54 & 57.13 & 55.08 & 55.90 & 58.68 & 55.37 \\
\hline 20 & 50.92 & 49.59 & 50.53 & 50.89 & 50.43 & 50.90 \\
\hline 21 & 44.79 & 40.04 & 41.18 & 41.57 & 41.07 & 41.59 \\
\hline 22 & 39.63 & 34.76 & 36.01 & 36.42 & 35.90 & 36.44 \\
\hline 23 & 44.83 & 43.42 & 44.41 & 44.73 & 44.21 & 44.76 \\
\hline 24 & 39.64 & 37.73 & 39.23 & 39.03 & 38.91 & 39.07 \\
\hline 25 & 36.38 & 34.07 & 35.98 & 35.34 & 35.56 & 35.40 \\
\hline 26 & 32.67 & 30.51 & 32.25 & 31.44 & 31.58 & 31.53 \\
\hline 27 & 31.66 & 30.32 & 31.20 & 30.15 & 30.22 & 30.29 \\
\hline 28 & 36.48 & 38.05 & 35.76 & 39.12 & 35.60 & 39.15 \\
\hline 29 & 32.04 & 30.08 & 31.06 & 30.21 & 30.94 & 30.26 \\
\hline 30 & 31.29 & 30.58 & 30.10 & 30.47 & 30.01 & 30.52 \\
\hline 31 & 31.81 & 30.90 & 30.58 & 30.75 & 30.13 & 30.80 \\
\hline 32 & 32.17 & 31.81 & 31.84 & 33.20 & 31.41 & 33.26 \\
\hline
\end{tabular}

TABLE XI

COST DATA FOR THE NEW YORK NETWORK

\begin{tabular}{llllll}
\hline No. & $\begin{array}{l}\text { Diameter } \\
\text { (in.) }\end{array}$ & $\begin{array}{l}\text { Cost } \\
(\$ / \text { feet })\end{array}$ & No. & $\begin{array}{l}\text { Diameter } \\
\text { (in.) }\end{array}$ & $\begin{array}{l}\text { Cost } \\
(\$ / \text { feet })\end{array}$ \\
\hline 1 & 0 & 0.00 & 9 & 120 & 417.0 \\
2 & 36 & 93.5 & 10 & 132 & 469.0 \\
3 & 48 & 134.0 & 11 & 144 & 522.0 \\
4 & 60 & 176.0 & 12 & 156 & 577.0 \\
5 & 72 & 221.0 & 13 & 168 & 632.0 \\
6 & 84 & 267.0 & 14 & 180 & 689.0 \\
7 & 96 & 316.0 & 15 & 192 & 746.0 \\
8 & 108 & 365.0 & 16 & 204 & 804.0 \\
\hline
\end{tabular}

20 runs are executed for both fixed and variable $p c$. Fig. 10 gives the iterative curves of two best solutions and changes in 20 runs for the New York problem with fixed and variable $p c$ respectively. We can find that the best solution is hit five times by the STA with fixed $p c$ and twice by the STA with variable $p c$.

Remark 5: Under the circumstance, the minimum function evaluations to achieve the best known solution is 5200, which takes up $2.6883 e-19 \%$ of all possible combinations $\left(16^{21}=\right.$ $1.9343 e 25)$.

Table XIII gives the best solutions gained by various algorithms, and it can be found that STA with both fixed and variable $p c$ can achieve the best known solution at the cost of 37.13 million dollars. As a matter of fact, the same solution was also gained by GA [9] with the function evaluations at $1,000,000$. The pressure heads for the New York network obtained by the discrete STA are given in Table XIV

\section{CONCLUSION}

The complexity of the water distribution network comes from two aspects, one is the linear and nonlinear equations, which are commonly handled by a hydraulic solver to ensure that the continuity and head loss equations are satisfied automatically, the other difficulty is that the commercial pipe size is discrete, which is proved to be NP-hard.

In this paper, It is shown that the network system can be reduced to the dimensionality of the number of closed simple loop or required independent paths, which can reduce 
TABLE XII

PIPE AND NODE DATA FOR THE NEW YORK NETWORK

\begin{tabular}{|c|c|c|c|c|c|}
\hline Pipe & Length (feet) ${ }^{4}$ & Existing Diameters (in.) & Node & Demand $\left(\text { feet }^{3} / \mathrm{s}\right)^{5}$ & Minimum Total Head (feet) \\
\hline 1 & 11600 & 180 & 1 & -2017.5 & 300.0 \\
\hline 2 & 19800 & 180 & 2 & 92.4 & 255.0 \\
\hline 3 & 7300 & 180 & 3 & 92.4 & 255.0 \\
\hline 4 & 8300 & 180 & 4 & 88.2 & 255.0 \\
\hline 5 & 8600 & 180 & 5 & 88.2 & 255.0 \\
\hline 6 & 19100 & 180 & 6 & 88.2 & 255.0 \\
\hline 7 & 9600 & 132 & 7 & 88.2 & 255.0 \\
\hline 8 & 12500 & 132 & 8 & 88.2 & 255.0 \\
\hline 9 & 9600 & 180 & 9 & 170.0 & 255.0 \\
\hline 10 & 11200 & 204 & 10 & 1.0 & 255.0 \\
\hline 11 & 14500 & 204 & 11 & 170.0 & 255.0 \\
\hline 12 & 12200 & 204 & 12 & 117.1 & 255.0 \\
\hline 13 & 24100 & 204 & 13 & 117.1 & 255.0 \\
\hline 14 & 21100 & 204 & 14 & 92.4 & 255.0 \\
\hline 15 & 15500 & 204 & 15 & 92.4 & 255.0 \\
\hline 16 & 26400 & 72 & 16 & 170.0 & 260.0 \\
\hline 17 & 31200 & 72 & 17 & 57.5 & 272.8 \\
\hline 18 & 24000 & 60 & 18 & 117.1 & 255.0 \\
\hline 19 & 14400 & 60 & 19 & 117.1 & 255.0 \\
\hline 20 & 38400 & 60 & 20 & 170.0 & 255.0 \\
\hline 21 & 26400 & 72 & - & - & - \\
\hline
\end{tabular}
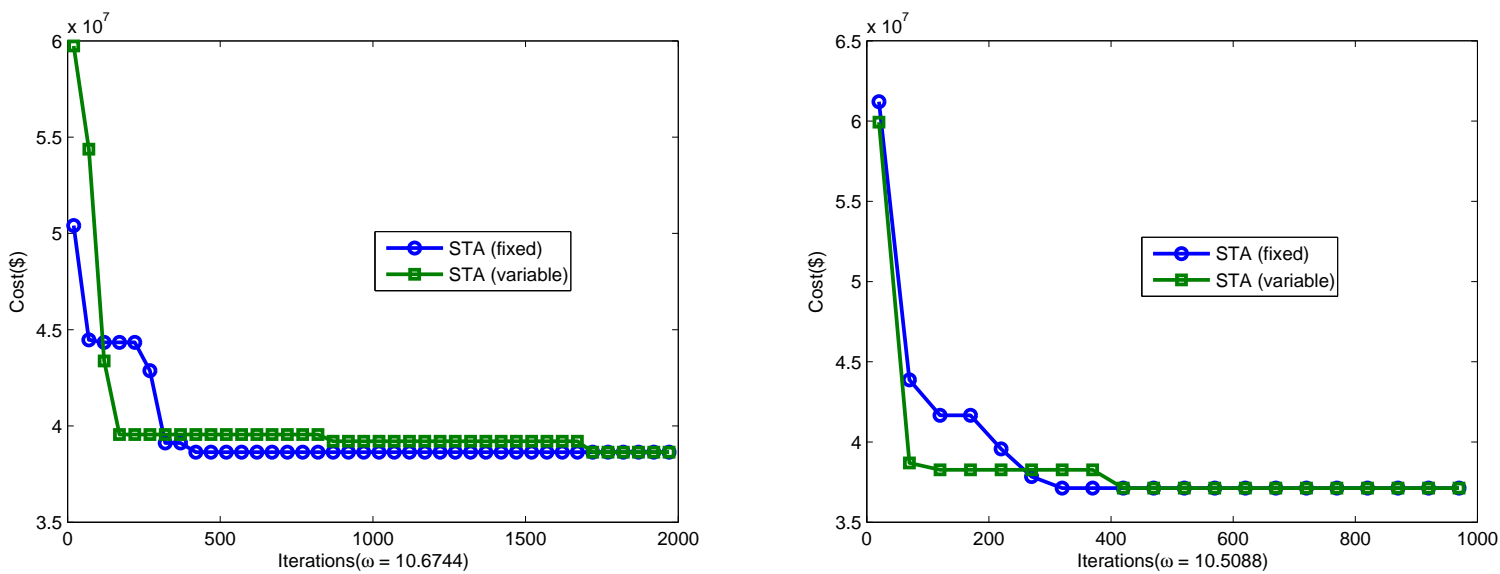

Fig. 10. Iterative curves of two best solutions using STA for the New York problem when $\omega$ is 10.6744 and 10.5088 , respectively

the computational complexity of solving linear and nonlinear equations simultaneously to a large extent.

To overcome the NP-hardness, a new intelligent optimization algorithm named discrete state transition algorithm is introduced to find the optimal or suboptimal solution. There are four intelligent operators in discrete STA, which are easy to understand and to be implemented. The "restore in probability" $p_{1}$ and "risk in probability" $p_{2}$ strategy in discrete STA is used to escape local optimal and increase the probability to capture the global optimum.

At first, a Monte Carlo simulation is studied to investigate a good combination of $p_{1}$ and $p_{2}$, and we find that $\left(p_{1}, p_{2}\right)=$ $(0.1,0.1)$ is a good choice. We then focus on a empirical study of the Two-Loop network, by training the network, we find that the penalty coefficient plays a significant role in the search ability and solution feasibility.

Based on the experience gained from the Two-Loop problem, the discrete STA has successfully applied to the Hanoi and New York networks, and the results show that the discrete STA can achieve the best known solutions with less function evaluations. The success of the discrete STA in optimal design of water distribution network has demonstrate that the discrete STA is a promising alternative in combinational optimization.

\section{ACKNOWLEDGMENT}

The authors would like to thank... 
TABLE XIII

SOLUTIONS FOR THE NEW YORK NETWORK

\begin{tabular}{lllllllll}
\hline Pipe & $\begin{array}{l}\text { Gessler } \\
{[2]}\end{array}$ & $\begin{array}{l}\text { Morgan and } \\
\text { Goulter [4] }\end{array}$ & $\begin{array}{l}\text { Dandy } \\
\text { et al. [8] }\end{array}$ & $\begin{array}{l}\text { STA } \\
\omega=10.6744\end{array}$ & $\omega=10.5088$ & $\begin{array}{l}\text { STA (variable) } \\
\omega=10.6744\end{array}$ & $\omega=10.5088$ \\
\hline 1 & 0 & 0 & 0 & 0 & 0 & 0 & 0 \\
2 & 0 & 0 & 0 & 0 & 0 & 0 & 0 \\
3 & 0 & 0 & 0 & 0 & 0 & 0 & 0 \\
4 & 0 & 0 & 0 & 0 & 0 & 0 & 0 \\
5 & 0 & 0 & 0 & 0 & 0 & 0 & 0 \\
6 & 0 & 0 & 0 & 0 & 0 & 0 & 0 \\
7 & 100 & 144 & 0 & 144 & 108 & 144 & 108 \\
8 & 100 & 144 & 0 & 0 & 0 & 0 & 0 \\
9 & 0 & 0 & 0 & 0 & 0 & 0 & 0 \\
10 & 0 & 0 & 0 & 0 & 0 & 0 & 0 \\
11 & 0 & 0 & 0 & 0 & 0 & 0 & 0 \\
12 & 0 & 0 & 0 & 0 & 0 & 0 & 0 \\
13 & 0 & 0 & 0 & 0 & 0 & 0 & 0 \\
14 & 0 & 0 & 0 & 0 & 0 & 0 & 0 \\
15 & 0 & 0 & 120 & 0 & 0 & 0 & 96 \\
16 & 100 & 96 & 84 & 96 & 96 & 96 & 72 \\
17 & 100 & 96 & 96 & 96 & 96 & 96 & 0 \\
18 & 80 & 84 & 84 & 84 & 96 & 84 & 72 \\
19 & 60 & 60 & 72 & 72 & 72 & 72 & 37.13 \\
20 & 0 & 0 & 0 & 0 & 0 & 0 & 72 \\
21 & 80 & 84 & 72 & 72 & 72 & 72 & 0 \\
Cost(\$ millions) & 41.80 & 39.20 & 38.80 & 38.64 & 37.13 & 38.64 & \\
\hline
\end{tabular}

TABLE XIV

Pressure HeAds For THE NeW York NeTWORK

\begin{tabular}{llllll}
\hline Node & STA & $\omega=10.5088$ & Node & $\begin{array}{l}\text { STA } \\
\omega=10.6744\end{array}$ & $\omega=10.5088$ \\
\hline 1 & 300.00 & 300.00 & 11 & 273.85 & 273.86 \\
2 & 294.20 & 294.33 & 12 & 275.12 & 275.15 \\
3 & 286.14 & 286.47 & 13 & 278.09 & 278.12 \\
4 & 283.78 & 284.16 & 14 & 285.55 & 285.58 \\
5 & 281.68 & 282.13 & 15 & 293.32 & 293.34 \\
6 & 280.06 & 280.55 & 16 & 260.05 & 260.16 \\
7 & 277.50 & 278.08 & 17 & 272.85 & 272.86 \\
8 & 276.65 & 276.51 & 18 & 261.15 & 261.30 \\
9 & 273.76 & 273.76 & 19 & 255.02 & 255.21 \\
10 & 273.73 & 273.73 & 20 & 260.70 & 260.81 \\
\hline
\end{tabular}

\section{REFERENCES}

[1] D. F. Yates, A. B. Templeman and T. B. Boffey, "The computational complexity of the problem of determining least capital cost designs for water supply networks," Engineering optimization, vol. 7, no. 2, pp. 142$155,1984$.

[2] J. Gessler, "Pipe network optimization by enumeration," Computer applications in water resources, ASCE, New York, pp. 572-581, 1985.

[3] E. Alperovits and U. Shamir, "Design of optimal water distribution systems," Water resources research, vol. 13, no. 6, pp. 885-900, 1977.

[4] D. R. Morgan and I.C. Goulter, "Optimal urban water distribution design," Water Resources Research, vol. 21, no. 5, pp. 642-652, 1985.

[5] I. C. Goulter, B. M. Lussier, and D. R. Morgan, "Implications of head loss path choice in the optimization", Water Resources Research, vol. 22, no. 5, pp. 819-822, 1986.

[6] A. Kessler and U. Shamir, "Analysis of the linear programming gradient method for optimal design of water supply networks", Water Resources Research, vol. 25, no. 7, pp. 1469-1480, 1989.

[7] G. Eiger, U. Shamir, and A. Ben-Tal, "Optimal design of water distribution networks", Water resources research, vol. 30, no. 9, pp. 2637-2646, 1994.
[8] G. C. Dandy, A. R. Simpson and L. J. Murphy, "An improved genetic algorithm for pipe network optimization", Water Resources Research, vol. 32, no. 2, pp. 449-458, 1996.

[9] D. A. Savic and G. A. Walters, "Genetic algorithms for least-cost design of water distribution networks", Journal of Water Resources Planning and Management, vol. 123, no. 2, pp. 67-77, 1997.

[10] A. R. Simpson, G. C. Dandy and L. J. Murphy, "Genetic algorithms compared to other techniques for pipe optimization", Journal of Water Resources Planning and Management, vol. 120, no. 4, pp. 423-443, 1994.

[11] M. da Conceição Cunha and J. Sousa, "Water distribution network design optimization: Simulated annealing approach", Journal of Water Resources Planning and Management, vol. 125, no. 4, pp. 215-221, 1999.

[12] S. Y. Liong and M. Atiquzzaman, "Optimal design of water distribution network using shuffled complex evolution", Journal of The Institution of Engineers, Singapore, vol. 44, no. 1, pp. 93-107, 2004.

[13] A. C. Zecchin, A. R. Simpson, H. R. Maier and J. B. Nixon, "Parametric study for an ant algorithm applied to water distribution system optimization", IEEE Transactions on Evolutionary Computation, vol. 9, no. 2, pp. 175-191, 2005.

[14] A. C. Zecchin, A.R. Simpson, H. R. Maier, M. Leonard, A. J. Roberts and M. J. Berrisford, "Application of two ant colony optimisation al- 


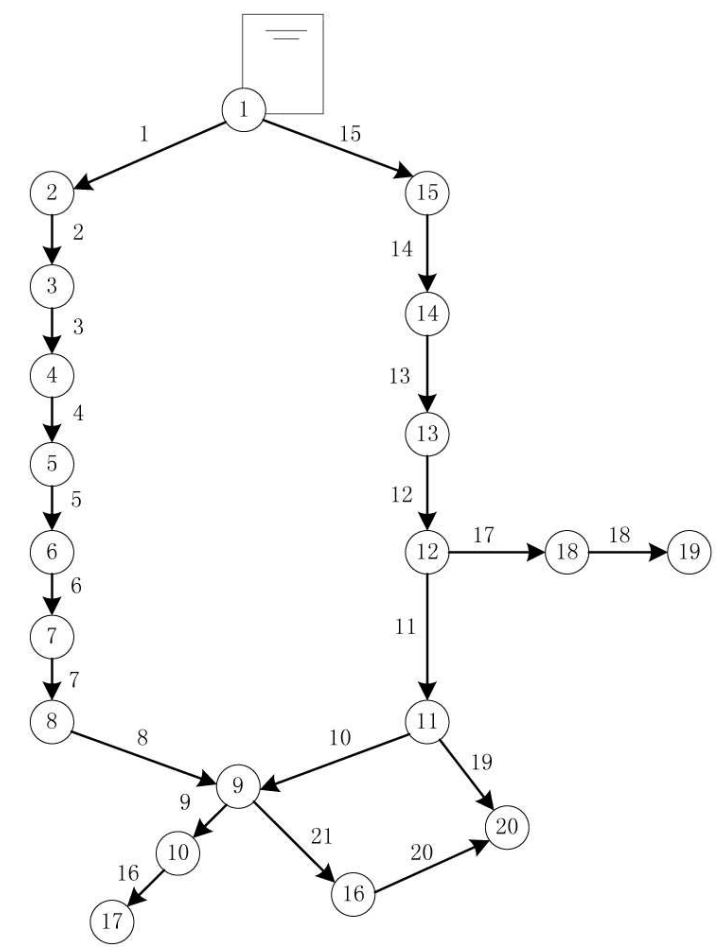

Fig. 9. the New York network

gorithms to water distribution system optimisation", Mathematical and computer modelling, vol. 44, no. 5, pp. 451-468, 2006.

[15] Z. W. Geem, "Optimal cost design of water distribution networks using harmony search", Engineering Optimization, vol. 38, no. 3, pp. 259-277, 2006.

[16] I. Montalvo, J. Izquierdo, R. Pérez, and M. M. Tung, "Particle Swarm Optimization applied to the design of water supply systems", Computers \& Mathematics with Applications, vol. 56, no. 3, pp. 769-776, 2008.

[17] A. Vasan and S. P. Simonovic, "Optimization of water distribution network design using differential evolution”, Journal of Water Resources Planning and Management, vol. 136, no. 2, pp. 279-287, 2010.

[18] M. Cisty, "Hybrid genetic algorithm and linear programming method for least-cost design of water distribution systems", Water resources management, vol. 24, no. 1, pp. 1-24, 2010.

[19] A. Haghighi, H. M. V. Samani and Z. M. V. Samani, "GA-ILP method for optimization of water distribution networks", Water resources management, vol. 25, no. 7, pp. 1791-1808, 2011.

[20] X. J. Zhou, C. H. Yang, W. H. Gui, "Initial version of state transition algorithm", in International conference on digital manufacturing and automation (ICDMA), 644-647, 2011.

[21] X. J. Zhou, C. H. Yang, W. H. Gui, "A new transformation into state transition algorithm for finding the global minimum", in International conference on intelligent control and information processing (ICICIP), 674-678, 2011.

[22] X. J. Zhou, C. H. Yang, W. H. Gui, "State transition algorithm", Journal of Industrial and Management Optimization, vol. 8, no. 4, pp. 1039-1056, 2012.

[23] C. H. Yang, X. L. Tang, X. J. Zhou and W. H. Gui, "State transition algorithm for traveling salesman problem", in Proceeding of the 31st Chinese Control Conference, pp. 2481-2485, 2012.

[24] Metropolis, N., Rosenbluth, A., Rosenbluth, M., Teller, A., Teller, E., Equation of state calculations by fast computing machines, Journal of Chemical Physics, 21(6), 1087-1092, 1953.

[25] Andradóttir, S., A global search method for discrete stochastic optimization, SIAM Journal on Optimization, 6(2), 513-530, 1996.

[26] Andradóttir, S., Accelerating the convergence of random search methods for discrete stochastic optimization, ACM Transactions on Modeling and Computer Simulation, 9(4), 349-380, 1999.

[27] Baba, N., Shoman T., Sawaragi Y., A modified convergence theorem for a random optimization method, Information Sciences, 13(2), 159-166, 1977.
[28] Van Laarhoven P.J.M., Aarts E.H.L., Simulated annealing: theory and applications, Dordrecht, The Netherlands, D. Reidel, 1987.

[29] Rossman, L.A., EPANET 2: users manual, US Environmental Protection Agency, Office of Research and Development, National Risk Management Research Laboratory, 1994.

[30] Wood, D.J., Computer Analysis of Flow in Pipe Networks Including Extended Period Simulations: User's Manual, Office of Continuing Education and Extension of the College of Engineering of the University of Kentucky, 1980. 